\title{
A Summary of 2018 and What Lies Ahead for Rheumatology and Therapy in 2019
}

Roy Fleischmann

Received: December 14, 2018 / Published online: January 5, 2019

(C) The Author(s) 2019

Keywords: Biosimilars; Gout; JAK inhibitors; Osteoarthritis; Psoriatic arthritis; Rheumatoid arthritis; Systemic lupus erythematosus

Dear Readers,

As 2018 comes to a close and we look forward to what is ahead within the field of rheumatology in 2019, we would like to say thank you to all of our authors, readers, reviewers, Editorial and Advisory boards, and everyone else who has contributed to Rheumatology and Therapy over the past 12 months.

In 2018, the readership of the journal was over 200,000 and the average citation rate was 2.4 citations per article. Some of the most highly read articles within 2018 included:

- Andrea Rubbert-Roth, Daniel E. Furst, Jan Michael Nebesky, Angela Jin, Erhan Berber. A review of recent advances using tocilizumab in the treatment of rheumatic diseases. Rheumatol Ther (2018)5:21. Over 6000 downloads and 5 citations to date, and an associated animated video summarizing the data.

- Peter Nash, Iain B. McInnes, Philip J. Mease, et al. Secukinumab versus adalimumab for psoriatic arthritis: comparative effectiveness

R. Fleischmann $(\bowtie)$

Metroplex Clinical Research Center, University of

Texas Southwestern Medical Center, Dallas, USA

e-mail: rfleischmann@arthdocs.com up to 48 weeks using a matching-adjusted indirect comparison. Rheumatol Ther (2018)5:99. 5800 downloads and 2 citations to date.

- Gergely Bodis, Victoria Toth, Andreas Schwarting. Role of human leukocyte antigens (HLA) in autoimmune diseases. Rheumatol Ther (2018)5:5. 4400 downloads and 2 citations to date.

- José Kennedy Amaral, Robert T. Schoen. A case report of chikungunya fever, rheumatoid arthritis, and Felty's Syndrome. Rheumatol Ther (2018)5:293. 4000 downloads to date.

We are also really pleased to be publishing more enhanced digital content such as an animated video explaining the process and rationale behind the use of a matched-adjusted indirect comparison of secukinumab and adalimumab for psoriatic arthritis, and a series of podcasts discussing the clinical challenges of oligoarticular psoriatic arthritis.

We have also begun publishing optional Plain Language Summaries alongside the standard scientific abstract to aid understanding and increase dissemination of research to nonexperts, including non-specialists, patients, caregivers, and others.

Looking ahead to 2019, we hope to see reports of many interesting and important developments in rheumatology. We should see results of important clinical trials of new JAK 
inhibitors in rheumatoid arthritis including the JAK1-specific inhibitors, filgotinib and upadacitinib, which may give us further valuable information as to where Jakinibs should be slotted in our therapeutic armamentarium; should they be used first line after a conventional synthetic DMARD, before a biologic DMARD, or after, or either? We may also see results of studies of Jakinibs in other autoimmune diseases such as psoriatic arthritis, ankylosing spondylitis, systemic lupus erythematosus, dermatomyositis, and inflammatory bowel disease, underscoring the importance of affecting intra-cellular signaling pathways in multiple autoimmune disease.

Multiple other medications are in development for rheumatoid arthritis including inhibitors of BTK, GM-CSF, CD40L, histone deacetylase 1, P2Y12, fractalkine, and alpha9 integrin; clinical results may be available in 2019, which may give us insight into whether any of these are of benefit in RA.

Similar efforts are underway in other diseases. In SLE, these include an anti-CD19 antibody Fc-engineered for increased affinity to FcgRIIb, low-dose IL2, an IgG1 $\kappa$ mAb, which blocks signaling of all IFNs, BTK inhibitors, IL12/23 mAb, JAK1/2, and COSL and BAFF bispecific inhibitors. In osteoarthritis, we should see results of clinical trials affecting pain (intraarticular CNTX-4975 [synthetic trans-capsaicin], subcutaneous tanezumab [humanized $\mathrm{mAb}$ that blocks nerve growth factor (NGF)], and an oral, peripheral-specific, pan-tropomyosin receptor kinase (Trk) inhibitor) and others affecting progression (Cathepsin $\mathrm{K}$ inhibitor, insulin-like growth factor 1 bone morphogenetic protein 7, Coll2-1 peptide, selective inhibitor of ADAMTS-5, and TPX-100 [peptide derived from matrix extracellular phosphoglycoprotein (MEPE)]). In scleroderma, where we are lacking effective medication for many aspects of disease, we may see reports on medications such as CBSO04, a mAb against Bdca-2, and lenabasum, a cannabinoid type 2 receptor agonist (which is also being evaluated in dermatomyositis). Molecules are in development for gout, including SEL-212, which is a pegadricase plus synthetic vaccine particles encapsulating rapamycin, which should limit antigenicity, NC-2700, a urate 1 inhibitor, ALLN-346, an oral urate oxidase targeting gut elimination of urate, and NC-2500, a xanthine oxidoreductase inhibitor.

In addition to reports of new molecules which describe their effectiveness and safety, we should also see further reports of the integrated safety analysis of compounds, such as the JAK inhibitors, which may clarify their risk with respect to venous thromboembolic events and whether there is a difference with respect to Jakinibs in terms of safety.

We expect that we will see significant improvement in study designs of molecules including improvement in assessment of endpoints so that appropriate endpoints are incorporated in studies and assessed appropriately. This will include endpoints in head-to-head studies in RA, which are vastly different compared to placebo-controlled studies, as well as clinically meaningful endpoints in SLE studies.

Very important to rheumatologists, patients, and society in general, is the answer to a very important question with respect to safety of biosimilar DMARDs (bsDMARDs). As many bsDMARDs are approved in many countries with effective registries, and as the choice of the bsDMARD may change week to week depending on cost, we may be able to determine if multiple switches between multiple bsDMARDs and the bio-original is effective clinically and safely without autoimmune events.

To summarize, Rheumatology and Therapy has had a successful 2018 and we are excited to see what develops in 2019.

Best wishes,

Roy Fleischmann, MD MACR.

\section{ACKNOWLEDGEMENTS}

This commentary is authored by the Editor-inChief of the journal and has not been peer reviewed.

Funding. No funding or sponsorship was received for this study or publication of this article. 
Authorship. All named authors meet the International Committee of Medical Journal Editors (ICMJE) criteria for authorship for this article, take responsibility for the integrity of the work as a whole, and have given their approval for this version to be published.

Disclosures. Roy Fleischmann has nothing to disclose.

Compliance with Ethics Guidelines. This article does not contain any studies with human participants or animals performed by any of the authors.
Open Access. This article is distributed under the terms of the Creative Commons Attribution-NonCommercial 4.0 International License (http://creativecommons.org/licenses/ by-nc/4.0/), which permits any noncommercial use, distribution, and reproduction in any medium, provided you give appropriate credit to the original author(s) and the source, provide a link to the Creative Commons license, and indicate if changes were made. 